\title{
LES ENJEUX D'UNE HISTOIRE DE LA PSYCHOLOGIE SOCIALE
}

Avant de présenter les lignes directrices selon lesquelles on peut envisager d'entreprendre une histoire de la psychologie sociale, je voudrais évoquer quelques-uns des problèmes qui se posent à son sujet et, plus généralement, à propos de l'histoire des sciences sociales.

POURQUOI SI PEU D'HISTOIRE?

Il suffit qu'un de ses membres émette le projet de rendre compte de l'histoire de sa discipline pour que la communauté scientifique qui se considère comme la gardienne légitime du savoir institué s'émeuve, s'inquiète et, réticente ou franchement hostile, propose de rejeter le transfuge hors de son sein. La suspicion qui accompagne celui qui manifeste de manière trop patente une curiosité pour sa propre histoire est un fait qui dépasse le cadre de la psychologie; elle touche, à des degrés divers, l'ensemble des sciences sociales'. La discussion qui a lieu, au sein des diverses commissions du C.N.R.S., sur le statut et le sort qui doit être réservé aux chercheurs dont l'activité s'est déplacée du "faire" de la psychologie (de la sociologie, de l'ethnologie...) au " réfléchir " sur les modalités de la constitution du savoir en psychologie (sociologie, etc.) est révélatrice et édifiante. L'argument généralement avancé pour justifier la mise à l'écart de ces cas embarrassants paraît à première vue légitime, et d'une objectivité sans faille apparente : pour leur propre confort, la place de ces chercheurs serait ailleurs, dans une commission spécialisée en histoire des sciences par exemple, composée de spécialistes qui seraient plus compétents pour évaluer un travail

\footnotetext{
1. Comme on a pu le constater à la lecture des rapports préparés, par discipline, pour le Colloque de définition sur l'histoire des sciences sociales et humaines en France qui s'est tenu sous l'égide du C.N.R.S. au printemps 1986.
}

Revue de synthèse: IV S. Nos 3-4, juil.-déc. 1988. 
historique et prodiguer des conseils, quand cela s'avère nécessaire. A y regarder de près, la proposition est spécieuse, ne serait-ce que parce que le chercheur, placé ainsi dans le statut inconfortable et précaire de " personne déplacée », perd toute possibilité de poursuivre simultanément ou de reprendre ultérieurement son activité initiale. Or, pour celui qui s'y engage, l'activité historique n'est en général pas une fin en soi mais plutôt un détour, une transition... De fait, l'enjeu réel est ailleurs. L'apparente objectivité de l'argumentation ne doit pas faire illusion : elle sert à masquer et à contourner la véritable question, celle des raisons pour lesquelles l'histoire fait peur. Le problème que l'on vient d'évoquer, se posant en des termes très similaires dans les autres institutions où le savoir s'élabore et dépassant largement les frontières de l'hexagone, mérite donc quelque réflexion.

Si l'histoire fait si peur, c'est qu'on reconnaît sans doute combien passé et présent ont partie liée. La façon dont on rend compte de celui-là engage celui-ci et la phrase de Lewis ${ }^{2}$, pour lapidaire qu'elle paraisse, comporte une.part de vérité : "Un nouveau futur exige un passé diffërent. " Chercher à rendre compte de l'histoire de sa discipline n'est donc jamais un acte gratuit, ni anodin, ni inoffensif. Il l'est d'autant moins que la discipline traverse à ce moment-là une crise paradigmatique ou épistémologique. Le fait que certains chercheurs abandonnent pour un temps le "faire " pour une activité réflexive est d'ailleurs, à cet égard, symptomatique; c'est rarement par un amour intrinsèque de l'histoire que l'on cherche à analyser et à connaître le passé de sá discipline mais plutôt pour des raisons qui tiennent à sa politique scientifique et/ou à ses options épistémologiques et qui engagent, par conséquent, directement son présent. En d'autres termes, la curiosité historique constitue inévitablement un défi pour l'identité présente de la discipline et, par ricochet, pour celle des chercheurs qui s'en réclament. Le commentaire de Lewis ${ }^{3}$, dans l'essai qu'il consacre à une réflexion sur l'activité d'historien, est pour une part transposable à notre propos. Prenant appui sur deux exemples, l'un ayant trait à la restauration récente de la commémoration de la bataille des Hébreux contre les Romains à Massada et au chapitre de l'histoire qui s'y rapporte, l'autre relatif à la restauration de la commémoration et de l'histoire de la fondation de l'État perse, il écrit :

2. Bernard LEWIS, History. Remembered, Recovered, Invented, Princeton, Princeton University Press, 1975, p. 11.

3. Ibid. 
«... dans l'un et l'autre cas, la restitution et la dissémination d'un chapitre oublié du passé est sous-tendue par un mobile idéologique puissant une forme nouvelle de prise de conscience, une tentative neuve de définir l'identité et les aspirations, et, par conséquent, l'obligation de dépasser ou d'approfondir l'héritage du passé, traditionnel et familier, mais relevant d'une image de soi discréditée ${ }^{4}$.

Pour en revenir à l'histoire des sciences, l'état du patrimoine d'une discipline $^{5}$ est l'aboutissement d'un développement singulier: les normes et les modalités qui la régissent, le résultat d'une sélection d'options théoriques, méthodologiques et épistémologiques particulières au détriment d'autres. Reconstituer le cheminement qui a abouti à ces choix successifs, mettre à nu les raisons qui ont abouti aux modes de fonctionnement actuels constituent une activité qui risque de troubler l'ordre régnant, de déstabiliser le statu quo sur lequel repose la vie de la communauté. Car, à moins qu'elle ne soit entreprise avec une visée explicitement apologétique comme justification du présent, la reconstitution historique a toutes les chances de révéler que la forme actuelle, instituée, de la discipline n'est ni nécessaire, ni inéluctable mais le produit de la convergence d'un ensemble de circonstances historiques et sociales particulières.

Si l'on ne peut que se soumettre à cette sanction de l'histoire, on est en droit de la juger plus ou moins subversive selon que l'on envisage d'un ceil plus ou moins favorable la mise en cause du statu quo et l'émergence, par le biais de ce retour en arrière, de nouveaux objets de recherche et de nouvelles orientations problématiques... Quoi qu'il en soit, l'activité historique est toujours dans un certain sens politique et engage une politique scientifique, en tous les cas quand elle est le fait des protagonistes de la discipline eux-mêmes ${ }^{6}$.

4. Ibid., p. 10, traduit par moi.

5. Cela est vrai également pour la mémoire collective qui le sous-tend, c'est-à-dire pour l'ensemble des éléments du passé transmis et entretenus par les efforts conscients des institutions du groupe. Cf. Maurice HalbWACHS, Les Cadres sociaux de la mémoire, Paris, P.U.F., 1952.

6. Cf. George Stocking, "On the Limits of 'Presentism' and 'Historicism' in the Historiography of the Behavioral Sciences ", Journal of the History of the Behavioral Sciences, t. 1, 3, 1965, p. 211-218. 


\section{LE POURQUOI ET LE COMMENT DE L'HISTOIRE}

Nous venons de suggérer qu'il y a diverses modalités pour organiser le passé et que la façon de faire est étroitement liée aux objectifs poursuivis, à la fonction assignée à l'histoire. Rappelons à cet égard les différences qui opposent les démarches caractéristiques, d'une part, de l'histoire rétrospective (presentist) et, d'autre part, de l'histoire historiciste (historicist) ${ }^{7}$. Dans la première, le passé est décodé à la lumière du présent, celui-ci servant en quelque sorte de schème d'intelligibilité à celui-là; dans la seconde, au contraire, le passé commande la lecture du passé puisque l'on tente, dans cette perspective, d'examiner l'émergence des théories successives à la lumière du contexte singulier qui leur donne naissance.

On conçoit assez aisément que la première démarche, tournée avant tout vers la recherche des origines par le biais d'une reconstitution des généalogies et des filiations des objets et des modalités de recherche actuels, ait avant tout une portée apologétique qui sert de justification au maintien de l'Establishment actuel et facilite la mise en place de mythes d'origine.

"Une histoire de la psychologie sociale n'a de raison d'être que s'il s'agit de trouver des justifications passées pour ce qui existe actuellement",

écrit Allport ${ }^{8}$, dans le chapitre qui fait fonction d'histoire officielle de la psychologie sociale depuis 1954 à ce jour (sans avoir subi de révisions au cours des rééditions successives), dans le manuel encyclopédique de la discipline. Explicite sur les fonctions qui doivent être celles de l'histoire, cette position annonce, sans aucune ambiguité pour qui connait les options théoriques de la psychologie sociale instituée contemporaine, quelle histoire nous est contée. Que cette histoire, dont l'auteur ne cache nullement les objectifs qu'il lui assigne (à savoir d'être une modalité de la reproduction des paradigmes existants), reste hautement sélective et tronquée est dès lors assez évident. Samelson ${ }^{9}$ analyse magistralement cette question mettant à jour la nature de la sélection des faits que l'auteur considère comme pertinents pour établir l'histoire officielle et les erreurs consécutives à une lecture dont la finalité est fixée $a$ priori; il fait apparaître ainsi quelques-unes des modalités

7. Ibid.

8. Gordon Allport, "The Historical Background of Modern Social Psychology ", in Gardner Lindzey, Elliott Aronson, eds, The Handbook of Social Psychology, Reading, Addison Wesley, 1954/1968, p. 1.80 , en part. p. 3.

9. Franz SAMELSON, "History, Origin Myth, and Ideology: Discovery of Social Psychology ", Journal for the Theory of Social Behavior, t. 4, 1974, p. 217-231. 
mises en cuvre dans une rétrospective qui a abouti à la création du mythe de l'origine positiviste de la discipline, mythe au nom duquel perdure un mode déterminé de production du savoir et se pratiquent aujourd'hui encore des exclusions.

Histoires rétrospective et historiciste ne supposent pas seulement des conceptions divergentes de l'histoire des sciences mais mettent simultanément en jeu des hypothèses distinctes sur l'évolution des théories dont chacune cherche à rendre compte. Une demarche historique qui consiste à remonter les filières et à reconstituer des filiations se fonde sur l'idée que le passé préfigure le développement ultérieur, que la science progresse selon un mouvement linéaire, soumise à un déterminisme purement interne, sans que l'on ait à se préoccuper des circonstances et du contexte dans lequel une théorie émerge. Une telle optique est congruente avec l'idée que la psychologie aurait pour vocation l'établissement de lois immuables et universelles des conduites humaines.

En revanche, dans une perspective historiciste, l'état de la science à un moment donné témoigne des questions qui traversent la période considérée en même temps que des réponses qu'elle y apporte, compte tenu de son état d'avancement. Une théorie dans cette optique est donc simultanément la manifestation de questions historiquement et sociologiquement situées et l'expression de la réponse que l'on tente d'y trouver. A l'inverse du précédent, ce point de vue suppose - ou du moins n'exclut pas - l'hypothèse du caractère transhistorique des objets de recherche, de leurs théories et modes d'approche; ainsi va-t-il à l'encontre d'une conception trop dogmatique de la science. A titre d'illustration, on peut citer, d'une part, le travail réalisé par Wood et Kröger ${ }^{10}$ et, d'autre part, celui de Danziger ${ }^{11}$ sur l'évolution de la conception de l'expérimentation. Les premiers auteurs montrent combien, chez Wundt, pourtant considéré communément comme le père de l'expérimentation moderne, la pratique de l'expérimentation était étrangère à celle qui est en vigueur de nos jours et, poursuivant plus loin cette idée, Danziger ${ }^{12}$ illustre plus largement le caractère transhistorique de la méthode expérimentale (posée en général comme strictement définie et immuable), en fonction notamment du type de question abordée et du type de population disponible pour les traiter.

10. Linda WOOD, Rolf KROGER, "Can we Revive the Classical Experiment for Social Psychology? ", Canadian Psychology, t. 26, 4, 1985, p. 282-291.

11. Kurt DANZIger, "The Origins of the Psychological Experiment as a Social Institution ", American Psychologist, t. 40, 2, 1985, p. 133-140.

12. Ibid. 


\section{CERNER L'OBJET D'UNE HISTOIRE DE LA PSYCHOLOGIE SOCIALE}

Préliminaire à tout travail historique, se pose la question de la délimitation de l'objet dont on fera l'histoire. $Y$ apporter une réponse est une tâche capitale, d'autant plus qu'elle engage la nature du travail ultérieur. Dans la discussion précédente sur les modalités d'organisation du passé, on a déjà pu entrevoir de quelle manière la détermination du corpus constitutif de la discipline engage des enjeux théoriques et épistémologiques. S'en tenir à ce qui paraît sous le label psychologie sociale (dans les revues et les ouvrages consacrés et reconnus par la communauté) revient à accepter implicitement comme pertinentes les options théoriques adoptées par cette communauté qui, contrỏlant l'accès des revues et des publications, exerce un droit de regard exclusif déterminant la légitimité de ce qui relève de la discipline. Accepter cette sélection comporte donc un biais dont les conséquences ont une portée épistémologique : elle conduit, notamment, à laisser pour compte toutes les tentatives, en particulier non abouties, qui traitent de la question du social sans s'aligner pour autant sur les normes de fonctionnement actuelles de la discipline, tout ce qui se constitue dans les marges du savoir officiel, formant ce que Netchine-Grynberg et Netchine ${ }^{13}$ désignent comme étant des " champs intermédiaires ". Or restituer ce qui est tombé dans l'oubli, ce qui a été écarté du patrimoine officiel constitue un travail, indispensable en lui-même, aussi bien qu'édifiant pour saisir la nature, les circonstances et la portée des choix des générations passées. En outre, ne pas considérer comme acquis et immuables les découpages notionnels et les clivages disciplinaires qui donnent leur physionomie actuelle au champ des sciences sociales ouvre sur des questions, tout à fait pertinentes pour notre propos, sur les enjeux et les modalités de la constitution même de champs disciplinaires distincts.

Pour toutes ces raisons et pour d'autres qui se dégageront dans la suite de cet exposé, il nous paraît préférable de faire l'histoire de la question $d u$ social plutôt que celle de la psychologie sociale, c'est-à-dire

13. Gaby NetChine-Grynberg, Serge Netchine, «A propos de quelques fictions dans la référence au biologique en psychologie : réduction ou formation de champs intermédiaires ", in La Fabrique, la figure et la feinte, éd. Paul MENGaL, Paris, Vrin, 1988, p. 81-101. 
de s'interroger sur la place que le social a occupée dans le développement des sciences humaines et sociales d'un point de vue théorique, même si la psychologie sociale n'existe, en tant que discipline autonome, reconnue sur le plan institutionnel, qu'à partir de la fin de la Seconde Guerre mondiale ${ }^{14}$. En se donnant cet objectif plus large, on contourne un premier obstacle épistémologique qui consisterait à cantonner l'histoire de la psychologie sociale exclusivement à ce qui se fait dans le cadre des frontières actuelles de la discipline instituée ${ }^{15}$. Cette façon de cerner l'objet de recherche a, en outre, comme avantage de considérer les frontières aussi bien que les options théoriques comme problématiques, comme faisant elles-mêmes partie intégrante de l'objet de recherche. Or, certainement à l'origine - mais cela reste vrai par la suite - , l'histoire des théories psychosociales, des pratiques psychosociologiques et celle de la psychologie sociale, envisagée comme discipline instituée, se recoupent sans se confondre les unes avec les autres ${ }^{16}$. Poser la question en termes plus larges parait donc heuristiquement plus approprié, notamment pour rendre compte de ce qui se passe à la fin du XIX siècle en France, où la question du social est présente dans tous les secteurs de la société et constitue une préoccupation majeure, tant dans la vie quotidienne que dans l'émergence des sciences sociales et humaines.

Publicistes, technocrates ou universitaires sont attentifs de diverses manières aux phénomèncs et aux facteurs sociaux au point que l'historien Hauser ${ }^{17}$, en 1903 , se croit fondé de parler de tarte à la crème à propos du social. Cela s'exprime dans les thèmes des théories politiques,

14. La première chaire de psychologie de la vie sociale a été créée en France en 1946 et c'est à la mème époque que sont mis en place, dans les universités américaines, les premiers programmes de PhD spécialisés en psychologie sociale.

15. On sera ainsi conduit à s'interroger, par exemple, sur les raisons qui ont consacré la fortune de l'épithète social dans le dernier tiers du $\mathrm{XIX}^{\mathbf{e}}$ siècle, raisons qui ont incité P. Chasles à choisir le titre Psychologie sociale des nouveaux peuples (1875) pour un ouvrage de linguistique comparée, tout à fait étranger à la psychologie sociale. On pourrait se demander en revanche pourquoi les éditeurs refusent que Tarde donne ce titre à l'ouvrage qui paraîtra sous l'intitulé Imitation sociale alors qu'il s'agit précisément d'un projet proprement psychosocial : cf. Ian LUBEK, « Histoire de psychologies sociales perdues : le cas de Gabriel Tarde ", Revue francaise de sociologie, t. 22, 1981, p. 361-395. On pourra encore relever et analyser dans quelles circonstances des durkheimiens, tels que Bouglé ou Lapie, n'ont pu faire mieux que de rester des adeptes clandestins de la psychologie sociale : cf. Erika APFElbaum, «Origines de la psychologie sociale. Développements souterrains et discipline méconnue ", Revue française de sociologie, t. 22, 1981, p. 397-407.

16. Le même type d'analyse est applicable à l'ensemble de la psychologie sociale avec des aspects spécifiques à chaque pays, mais dans la suite nous traiterons avant tout de la conjoncture telle qu'elle existe en France.

17. Henri HAUSER, L'Enseignement des sciences sociales. État actuel de cet enseignement dans les divers pays du monde, Paris, Chevalier-Marescq, 1903. 
collectivistes ou solidaristes, se traduit dans la volonté de développer une pratique destinée à gérer les questions sociales (pallier au paupérisme et à la misère sociale) mais marque aussi la psychologie naissante $^{18}$.

La position de la problématique sociale est paradoxale puisqu'elle est tout à la fois omniprésente et absente, la psychologie sociale n'ayant aucune existence institutionnelle autonome au sein de l'Université au moment où les autres disciplines, et notamment la psychologie et la sociologie, y gagnent leurs lettres de noblesse.

La problématique sociale est présente parce que, sans pour autant vouloir instituer un autre mythe d'origine, c'est à certains égards en France que naissent, à cette époque, les premières théories importantes et systématiques de psychologie sociale. Entre les années 1880 et 1900 , on voit fleurir des cours de psychologie sociale dans les diverses institutions parallèles qui existent à la périphérie de la Sorbonne. Presque simultanément, Hamon, Tarde, Le Bon et Binet proposent des théories des conduites sociales interpersonnelles ou collectives. Un an avant sa mort en 1911, Binet plaidera même tout à fait explicitement pour le développement d'une psychologie sociale, discipline autonome au même titre que la psychologie ${ }^{19}$. Sans véritable prolongement en France où ils tombent vite dans l'oubli, ces premiers écrits psychosociaux ont en revanche laissé une empreinte sur la psychologie sociale américaine naissante du début du $\mathrm{XX}^{\mathrm{e}}$ siècle - même si ces sources sont aujourd'hui méconnues jusque dans les essais historiques français ${ }^{20}$.

Présente encore parce que la conception du psychologique a constamment été conçue, davantage en France que dans d'autres pays, en relation étroite avec celle du social. Cela est manifeste dans les deux domaines dont la psychologie française fait à l'origine ses terrains d'élection, la psychopathologie et la psychopédagogie. A la fin du XIX siècle, dans l'affrontement qui oppose, sur un terrain particulièrement sensible de la psychopathologie, celle de la question de l'hystérie, les équipes rivales de Bernheim à Nancy et de Charcot à La Salpêtrière, l'enjeu central du débat porte sur le statut de la question du relationnel dans les conceptions modernes de la pathologie ${ }^{21}$. La victoire de la position

18. Cf. mon article, E. Apfelbaum, « Prolegomena for a History of Social Psychology : Some Hypotheses Concerning its Emergence in the Twentieth Century and its Raison d'ètre $"$, in Knud LARSEN, ed., Psychology and Ideology, New York, Ablex, 1986, t. 2, p. 3-13.

19. Cf. art. cit. supra n. 15.

20. Cf. op. cit. supra n. 8, 1968.

21. E. ApFelbaum, "Entre la volonté et la contrainte: de l'hypnose à l'influence, l'histoire d'un évitement ", in Sacha BEM, Hans RAPPARD, Wilhem VAN HoORN, eds, Studies 
de Bernheim et le ralliement de Binet constituent un pas en faveur d'une conception qui reconnaît l'importance du relationnel. C'est d'ailleurs en prenant en compte la composante relationnelle que la psychologie établit et délimite un terrain autonome distinct de celui qui lui est assigné jusque-là - la théorie de la suggestibilité ${ }^{22}$, forme primitive d'une théorie de l'influence sociale, en est un des exemples. D'autre part, en ce qui concerne le terrain de la psychologie de l'enfant et de la pédagogie, ces disciplines naissent des préoccupations qui proviennent de l'instauration de la scolarité obligatoire et du besoin de gérer efficacement les mouvements de masses importantes d'écoliers d'origines diverses et de niveau scolaire très hétérogène. L'importance des dimensions sociales est très vite reconnue par Binet qui voit dans les différences de "milieu social " l'explication des différences qu'il observe dans les conditions physiques des écoliers, leur fatigue, leurs motivations et leur réussite scolaire ${ }^{23}$.

Plus généralement, on peut souligner que, sur le plan théorique, la référence au social dans la psychologie appartient en propre à une tradition française et l'on en retrouve la trace tout au long de la première moitié du $\mathrm{XX}^{e}$ siècle aussi bien chez Lagache que chez Janet. Par ailleurs, cette tradition est enracinée avant tout dans la tradition sociologique durkheimienne: s'il y a bien chez Binet toute une thématique de l'influence du social dans le développement individuel, elle fait avant tout référence au milieu social; l'étude du lien social est, en revanche, dans une large mesure méconnue. Tout se passe comme si le poids de la tradition sociologique française avait fait obstacle à l'avènement d'une discipline psychosociale autonome. Ni le plaidoyer de Binet en faveur de la psychologie sociale, ni la défense de Bouglé ne furent entendus en leur temps; de sorte que faire l'histoire de la psychologie sociale en France dans la première moitié du $\mathrm{xx}^{e}$ siècle revient à faire celle des raisons qui ont fait qu'il n'y a pas eu de psychologie sociale française à proprement parler, à faire aussi l'histoire de ce qui en a occupé la place ou en a tenu lieu. A certains égards, la psychanalyse, en traitant du registre du relationnel, a elle aussi fait obstacle dans la mesure où elle pouvait prétendre occuper cet autre terrain dont se réclame la psychologie sociale.

in the History of Psychology and the Social Sciences, Proceedings of the Third European Meeting of Cheiron, Leiden, Leiden Psychological Institute, 1985, p. 95-108.

22. Alfred Binet, La Suggestibilité, Paris, Schleicher, 1900.

23. A. Binet, Théodore Simon, "La misère physiologique et la misère sociale ", Année psychologique, t. 12, 1906, p. 1.24. 
Au lendemain de la Seconde Guerre mondiale, les questions pertinentes à l'intelligibilité du développement de la psychologie sociale changent sensiblement de nature. Reconnue sur le plan institutionnel, la création d'une chaire de psychologie de la vie sociale consacre la reconnaissance de la discipline au sein de la Sorbonne; la recherche en psychologie sociale se développe au C.N.R.S., en même temps que celle de la psychologie et de la sociologie. Mais, à la suite de ce changement de statut, l'évolution de la discipline devient plus étroitement tributaire de la politique de ses institutions de tutelle. L'analyse des contraintes et des obligations qu'elles lui imposent devient par conséquent plus essentielle à ce stade de son développement; en ce qui concerne la France, il convient d'accorder une attention particulière aux circonstances qui ont abouti à de nouvelles lignes de partage ainsi qu'aux modifications dans la répartition entre les différents secteurs du C.N.R.S. que les diverses disciplines des sciences sociales et humaines ont connues. Le fait que la psychologie sociale soit rattachée avec la psychologie, depuis les années 1960 , au secteur des sciences de la vie - cette séparation, voire cette opposition, institutionnelle instaurant des frontières de fait entre ces différents domaines des sciences sociales, notamment avec la sociologie ou l'anthropologie - a été un des facteurs déterminants pour l'évolution de sa problématique et de ses objets de recherche.

En France encore, le hiatus qui existe souvent, sur le plan de son contenu, entre la psychologie sociale qui se développe au C.N.R.S. et celle qui est enseignée dans les universités traduit l'étanchéité des barrières qui peuvent séparer, dans de nombreux cas, institutions d'enseignement et de recherche et le manque de circulation des idées qui en résulte. Cet état de choses est caractéristique de la conjoncture institutionnelle française et doit être pris en compte si l'on veut comprendre certaines des manifestations singulières de l'évolution de la discipline dans $c$ pays.

Enfin, sans pour autant prétendre à l'exhaustivité, la coupure qui s'est progressivement instaurée entre la psychologie sociale et la psychosociologie est une autre composante du paysage français, capitale pour comprendre la physionomie particulière affichée ici par les conceptions du social. Psychologie sociale et psychosociologie renvoient à deux conceptions distinctes d'une problématique du social, qui coexistaient à l'origine de façon complémentaire; l'un des enjeux de leur divorce est à chercher, d'une part, dans la hiérarchisation de statut implicitement établi entre une science qui viserait des processus universels et celle qui reste en prise avec la réalité sociale et, d'autre part, dans la politique 
qui prévalait dans les années 1960 concernant les contrats avec les organismes privés de financement de la recherche. Désormais, il s'agit de deux orientations aux visées distinctes, entre lesquelles il y a peu de points communs : la psychologie sociale, se donnant comme étude des processus fondamentaux, se fonde principalement sur une approche expérimentale et se développe - de façon d'ailleurs relativement limitée - au sein du C.N.R.S., tandis que la psychosociologie, centrée sur l'étude des groupes et des relations interpersonnelles s'épanouit largement mais principalement hors de l'institution universitaire. Ce clivage recoupe partiellement celui qui tend à opposer une recherche qui se veut fondamentale à celle qui reste tournée vers la réalité sociale; sur ce dernier point, la situation, très différente de celle qui prévaut tant en Amérique du Nord qu'en Grande-Bretagne où théorie et pratiques sociales s'enrichissent mutuellement, a donné naissance à une communauté scientifique dont la vitalité et la résistance semblent beaucoup plus assurées que les nôtres.

Toutefois, le facteur le plus déterminant pour comprendre les options, théoriques et méthodologiques, qui caractérisent la psychologie sociale dans la seconde moitié du $\mathrm{XX}^{e}$ siècle par-delà la diversité de ses destinées nationales, reste l'importance de la compétition scientifique internationale qui déplace le centre de gravité des sources d'influence. Les sociétés savantes jouent désormais un rôle capital dans le processus de normalisation aussi bien des problématiques que des modes de production des connaissances scientifiques. Le rapport de force qui existe actuellement en faveur de la psychologie sociale américaine pèse lourdement sur les destinées des sujets traités par la psychologie sociale en France. C'est donc également dans l'histoire de la psychologie et de la psychologie sociale américaine qu'il faut rechercher, en partie du moins, l'origine et les raisons des conceptions contemporaines de la psychologie sociale et de ses normes de fonctionnement scientifique.

Autrement dit, jusqu'au milieu du $\mathrm{Xx}^{e}$ siècle, dans la période initiale du développement de la discipline, antérieure donc à sa phase de normalisation, une histoire du social doit rendre compte des destinées nationales de la discipline (notamment en ce qui concerne ses manifestations en Allemagne, aux États-Unis et en France). Pour ce dernier pays, où existe une tradition substantielle de la pensée sociale, il conviendra d'en dégager la spécificité, tout en rendant compte des causes de l'éclipse totale que la psychologie sociale a connue après la mort de Binet et jusqu'à la création d'une licence de psychologie après 1945 . La singularité de la conjoncture théorique et pratique française - l'importance de l'épistémologie, du marxisme, du structuralisme et de la psychanalyse 
- n'est sans doute pas totalement étrangère à la trajectoire particulière qu'a connue la discipline dans ce pays.

Ce n'est que dans la période contemporaine que l'on peut parler de psychologie sociale à proprement parler comme discipline homogène et unifiée et, dès lors, à la suite de la migration des chercheurs d'Europe dans les années $1930^{24}$ et sous l'impulsion donnée à la discipline par l' "effort de guerre " aux États-Unis, le centre de gravité se déplace et son devenir se décide désormais pour une large part outre-atlantique.

Voici en quels termes il me paraîtrait heuristiquement pertinent de poser l'objet problématique d'une histoire de la psychologie sociale dans laquelle sont étroitement imbriquées déterminations internes et externes, évolution des institutions et histoire des idées. C'est simultanément sur ces diffërents plans qu'il faut rendre compte de cette histoire du social et des rapports conflictuels que la psychologie sociale entretient avec les autres domaines des sciences sociales. D'ailleurs, la question du social traversant l'ensemble des sciences sociales et humaines, retracer l'évolution de son statut épistémologique est importante pour comprendre le développement de la psychologie sociale en France (par comparaison avec celui qu'il a connu dans les pays anglo-saxons); elle ne l'est pas moins pour rendre compte de celui de la psychologie et de la sociologie et des rapports qui lient ces disciplines entre elles. Elle leur sert en quelque sorte d'analyseur.

Erika APFElBAUM, GEDISST, C.N.R.S. ${ }^{2 S}$

24. H. Stuart Hughes, The Sea Change: The Migration of Social Thought, 1930-1965, New York, Mc Graw Hill, 1975.

25. Institut de recherche sur les sociétés contemporaines, 59 rue Pouchet, 75849 Paris Cedex 17. 